\section{L'Actualité économique}

L'ACTUALITÉ ÉCONOMIQUE

Managerial Comparisons of Four Developed Countries: REVUE D'ANALYSE ÉCONOMIQUE

France, Britain, United States and Russia, par DAVID GRANICK. — THE MIT PRESS, 1972.

\title{
André Joyal
}

Volume 52, numéro 3, juillet-septembre 1976

URI : https://id.erudit.org/iderudit/800689ar

DOI : https://doi.org/10.7202/800689ar

Aller au sommaire du numéro

Éditeur(s)

HEC Montréal

ISSN

0001-771X (imprimé)

1710-3991 (numérique)

Découvrir la revue

Citer ce compte rendu

Joyal, A. (1976). Compte rendu de [Managerial Comparisons of Four Developed Countries: France, Britain, United States and Russia, par DAVID GRANICK. THE MIT PRESS, 1972.] L'Actualité économique, 52(3), 406-408.

https://doi.org/10.7202/800689ar d'utilisation que vous pouvez consulter en ligne.

https://apropos.erudit.org/fr/usagers/politique-dutilisation/ 


\section{Managerial Comparisons of Four Developed Countries: France, Britain, United States and Russia, par DAvID GranICK. - THE MIT PRESS, 1972.}

Dans cet ouvrage, l'auteur tente de comparer les performances et le comportement des dirigeants d'entreprises en s'appuyant sur l'hypothès que des distinctions significatives peuvent être mises en évidence entre le modal managerial behavior de quatre pays industrialisés mais différents par leurs traditions et leurs contextes historique et politique. En considérant que les attitudes des administrateurs d'entreprises sont, d'une part, influencées par le système de valeurs du milieu environnant et, d'autre part, par la formation, la sélection et les stimulants économiques, il s'agit de voir l'effet de ces variables sur les performances des entreprises et, par la suite, sur l'évolution de la productivité industrielle.

Les informations recueillies ont été obtenues à la suite d'interviews effectuées auprès d'une dizaine d'entreprises en France et en Angleterre et auprès d'un nombre équivalent aux Etats-Unis. Les informations sur le fonctionnement des entreprises soviétiques ont été recueillies à partir de la documentation officielle ainsi que par les contacts établis à la faveur d'un court séjour dans ce pays. L'importance accordée à l'URSS est 
marginale par rapport à l'attention accordée à l'administration des firmes françaises et anglaises. On cherche en vain une allusion à l'URSS dans la table des matières. Le lecteur intéressé doit se rapporter aux chapitres 7 et 9 traitant respectivement de la sélection des managers et des stimulants économiques où 18 pages sont consacrées à ce qui prévaut en la matière en URSS.

Le petit nombre d'entreprises constituant l'échantillon ne doit pas éveiller outre mesure les doutes quant à sa représentativité. Un chapitre entier (chap. 4) est consacré à la justification du choix des entreprises retenues. Que ce soit par leurs performances, leur dimension et leur champ d'activité, l'auteur explique de façon détaillée les critères qui ont guidé son choix. Chacune des entreprises retenues dans les trois pays occidentaux fait partie des deux cents plus grandes entreprises de chacun des pays. Pour la France et l'Angleterre, seules les entreprises privées font partie de l'échantillon étudié. Ainsi, les lecteurs intéressés par la problématique de la petite et moyenne entreprise ne trouveront pas, dans cette étude, des éléments susceptibles de correspondre à leurs préoccupations.

Il faut mentionner, cependant, que l'auteur a pris soin de distinguer quatre types de lecteurs pouvant être intéressés par sa recherche. Il spécifie les chapitres qui devraient correspondre aux intérêts de différentes catégories de spécialistes. Sur les quatorze chapitres, seulement six devraient bénéficier de l'attention particulière des économistes. Ce sont le chapitre 1, où les fondements théoriques de l'étude sont décrits, et le chapitre 4, déjà signalé. Les quatre autres chapitres destinés aux disciples d'Adam Smith se rapportent aux différents aspects de la carrière des managers et aux politiques d'investissement et de prix. Les autres catégories de lecteurs à qui cet ouvrage est destiné sont les spécialistes de la sociologie industrielle, de l'administration des affaires et tous ceux que la croissance économique en général intéresse.

Les conséquences du système d'éducation français qui font que les administrateurs haut placés au sein des entreprises importantes sont tous issus des «grandes écoles» sont fort bien montrées. Le très grand contraste dans le management français et anglais est en partie expliqué par le prestige de la fonction qui est beaucoup plus grand en France. L'auteur y voit une des explications de la plus grande efficacité des entreprises françaises qui, en dépit du fait que leur dimension soit inférieure à celle de leurs homologues d'outre-manche, affichent une plus grande productivité. Le système de «promotion ouvert 》 qui prévaut en Angleterre est une distinction importante par rapport au système français considéré comme étant «fermé ». A l'appui de ceci on a déjà cité l'exemple d'une compagnie minière dont les cadres supérieurs et moyens, sans exception, étaient tous issus de l'Ecole des Mines de Paris. En opposition, l'auteur signale également la forte mobilité qui caractérise le personnel de gestion des entreprises américaines. Ce qui, pour lui, aurait l'inconvénient d'exiger le recours à des efforts répétés d'initiation aux nouvelles fonctions administratives. Or, il est à se demander ici si cet inconvénient ne peut être compensé par l'expérience acquise chez un ou plusieurs concurrents. Quant 
à la distinction dans le comportement des managers soviétiques par rapport à leurs homologues américains, l'auteur signale, comme on pouvait s'y attendre, la célèbre tendance qu'ont les premiers à sous-estimer la capacité productive de leur entreprise afin de s'assurer une plus grande part de revenu sous forme de primes.

En somme, tous ceux qui sont intéressés par le fonctionnement des grandes entreprises principalement en France et en Angleterre trouveront dans cet ouvrage une foule d'informations permettant de mieux comprendre les hauts et les bas de certains secteurs d'activités de ces deux économies.

André JOYAL, Université du Québec d Trois-Rivières 